\title{
Prevalence and antibiotic resistance of Acinetobacter baumannii among patients in postcardiac surgery intensive care units of Rajaei Hospital, Tehran
}

\author{
Monireh Kamali ${ }^{1}$, Shirin Manshouri ${ }^{1}$, Yasser Bagheri ${ }^{2,3}$, Masoumeh Rostami ${ }^{1}$, Mahdi Karkhaneh Mahmoudi ${ }^{1}$, \\ Pardis Moradnejad ${ }^{1}$, Farhad Seif*4,5(1)
}

Received: 15 Jun 2019

Published: 11 Feb 2020

\section{Abstract}

Background: Acinetobacter baumannii is an opportunistic, aerobic, nonfermentative, Gram-negative bacterium that can cause major nosocomial infections, especially in patients hospitalized in intensive care units (ICU). Recently, A. baumannii strains have been resistant to a variety of antibiotics. Thus, it was aimed to evaluate the prevalence of $A$. baumannii and their resistance to the antibiotics in the patients hospitalized in postcardiac surgery ICU.

Methods: This retrospective cross sectional study was performed in Rajaei hospital between March 2014 and February 2016 . $A$. baumannii strains were isolated from blood cultures, catheter cultures, sputum cultures, and wound smear cultures. Then, isolates were characterized using standard morphological, cultural, and biochemical properties according to CLSI 2016. The frequency of A. baumannii species were reported as percent.

Results: Among 27167 patients were admitted to the ICU, 113 individuals, including 55 males and 58 females, were identified as $A$. baumannii-infected and the prevalence rate was $0.42 \%$. The highest rates of antibiotic sensitivity were related to Meropenem 20 $(17.7 \%)$ and Colistin $16(14.1 \%)$. The shortest length of stay (LOS) for patients with A. baumannii in the ICU was 3 days, while the longest LOS was 98 days.

Conclusion: The findings indicated that $A$. baumannii strains isolated from postcardiac surgery ICUs had a high prevalence and were sensitive to Meropenem and Colistin. However, new molecular-based techniques are needed to monitor nosocomial infections. Therefore, the treatment of the patients may be feasible by appropriate antibiotic therapy, and infection control policies will be improved by adopting precise disinfection strategies.

Keywords: Acinetobacter baumannii, Nosocomial infection, Antibiotic, Resistance

Conflicts of Interest: None declared

Funding: None

\section{*This work has been published under CC BY-NC-SA 1.0 license.}

Copyright $\subseteq$ Iran University of Medical Sciences

Cite this article as: Kamali M, Manshouri Sh, Bagheri Y, Rostami M, Karkhaneh Mahmoudi M, Moradnejad P, Seif F. Prevalence and antibiotic resistance of Acinetobacter baumannii among patients in postcardiac surgery intensive care units of Rajaei Hospital, Tehran. Med J Islam Repub Iran 2020 (11 Feb);34:4. https://doi.org/10.47176/mjiri.34.4

\section{Introduction}

Antibiotic resistance is a global problem threatening public health. The outcomes of the antibiotic-resistant

Corresponding author: Dr Farhad Seif, seif.f@tak.iums.ac.ir

1. Rajaei Cardiovascular Medical and Research Center, Iran University of Medical Sciences, Tehran, Iran

2. Immunology Research Center, Institute of Immunology and infectious Diseases, Iran University of Medical Sciences, Tehran, Iran

3. Department of Immunology, School of Medicine, Iran University of Medical Sciences, Tehran, Iran

4. Neuroscience Research Center, Iran University of Medical Sciences, Tehran, Iran

5. Academic Center for Education, Culture, and Research, Tehran, Iran infections are longer hospitalization, higher cost of medical health care, and increased morbidity and

$\uparrow$ What is "already known" in this topic:

Acinetobacter baumannii is an aerobic, nonfermentative, Gram-negative microorganism, causing major nosocomial infections, especially in patients hospitalized in intensive care units (ICU). The prevalence of $A$. baumannii and its resistance to antibiotics may promote health conditions and save patients' lives.

\section{$\rightarrow$ What this article adds:}

The high prevalence and, on the other hand, the sensitivity of A. baumannii to Meropenem and Colistin are notable to treat patients in a more targeted manner and provide new disinfection approaches. 
mortality (1). Acinetobacter baumannii is an opportunistic, aero bic, nonfermentative, and Gramnegative bacterium that can cause major nosocomial infections in seriously ill patients with broad-spectrum antibiotics who are hospitalized in intensive care units (ICUs) (1-3). The mortality rate is variable and ranges from $17 \%$ to $63 \%$ (4). A. baumannii can cause bacteremia through the bloodstream, pneumonia, septic shock, ventilator-associated pneumonia, disseminated intravascular coagulation (DIC), and in patients who suffer from burns or urinary tract infections (UTI); the inappropriate selection of antibiotics leads to poor clinical outcomes and increased mortality rates (5-7).

Recently, A. baumannii strains have been resistant to the family of $\beta$-lactam antibiotics such as Carbapenems and have become multidrug-resistant (MDR) pathogens (8). However, Aminoglycosides, Tigecycline, Colistin (polymyxin E), Sulbactam, and Minocycline have shown limited therapeutic success (9). Since MDR-resistant $A$. baumannii isolates are frequently found in nosocomial infections in patients requiring serious health care such as postcardiac surgery ICU (10-12); thus, this study aimed to evaluate the prevalence of $A$. baumannii and their resistance to antibiotics in patients who were hospitalized in postcardiac surgery ICU.

\section{Methods}

This retrospective cross sectional study was performed in Rajaei hospital, Tehran, Iran. A total of 27167 patients admitted to the ICU between March 2014 and February 2016 and patients who had positive culture results for (MDR) A. baumannii from any specimen (blood, sputum, urine, body fluid, or wound) were included in the study. The criteria for clinical isolates were based on the CDC definition for nosocomial infections (13).

Isolation, characterization, and susceptibility assessment: The specimens collected were blood cultures, catheters, sputum, wound smear, etc. Isolation of the bacteria was done by culturing on either selective agar media (Blood Agar, MacConkey agar, and Chocolate Agar) or enriched media (Thioglycolate) at $37^{\circ} \mathrm{C}$ for 24 to 48 hours (14). Methylene blue and Gram staining, oxidase test, and other differential biochemical tests were also done (15). Clinical isolates underwent species determination, and antibiotic susceptibility testing was performed by Vitek-2 system (bioMérieux, Marcy l'Etoile, France) by means of IDGN and N090 panels.

Antimicrobial susceptibility was determined using the disk diffusion technique, in accordance with the criteria established by the Clinical and Laboratory Standards Institute (CLSI) 2016 (16). The E-test method was used to assess the antimicrobial susceptibility of $A$. baumannii isolates (17). A. baumannii was considered MDR if it was resistant to routinely used antibiotics (including Penicillin, Cephalosporins, Fluoroquinolones, Aminoglycosides, or Trimethoprim-sulfamethoxazole) but susceptible to Carbapenems (18).

The demographic characteristics of the patients were obtained from clinical records. This information included gender, age, clinical diagnosis, the use of medical devices, comorbidities, reason for ICU admission, organ dysfunction at ICU admission, ICU length of stay (LOS), and consciousness level. Patients with evidence of any concurrent bacterial infection and children were excluded from this study. All analyses were performed using SPSS, version 20 (SPSS Inc., Chicago, Illinois, USA). All tests were 2-tailed. The normality of the data was evaluated by Kolmogorov-Smirnov test, and $\mathrm{p}<0.05$ was considered to be statistically significant (19).

\section{Results}

Among 27167 patients admitted to ICU, 113 individuals (55 males and 58 females) were identified as MDR A. baumannii infected, with the prevalence rate of $0.42 \%$. Mean admission time before the diagnosis of MDR A. baumannii for different wards of the ICU was determined (Fig. 1).

The shortest LOS for patients with (MDR) $A$.

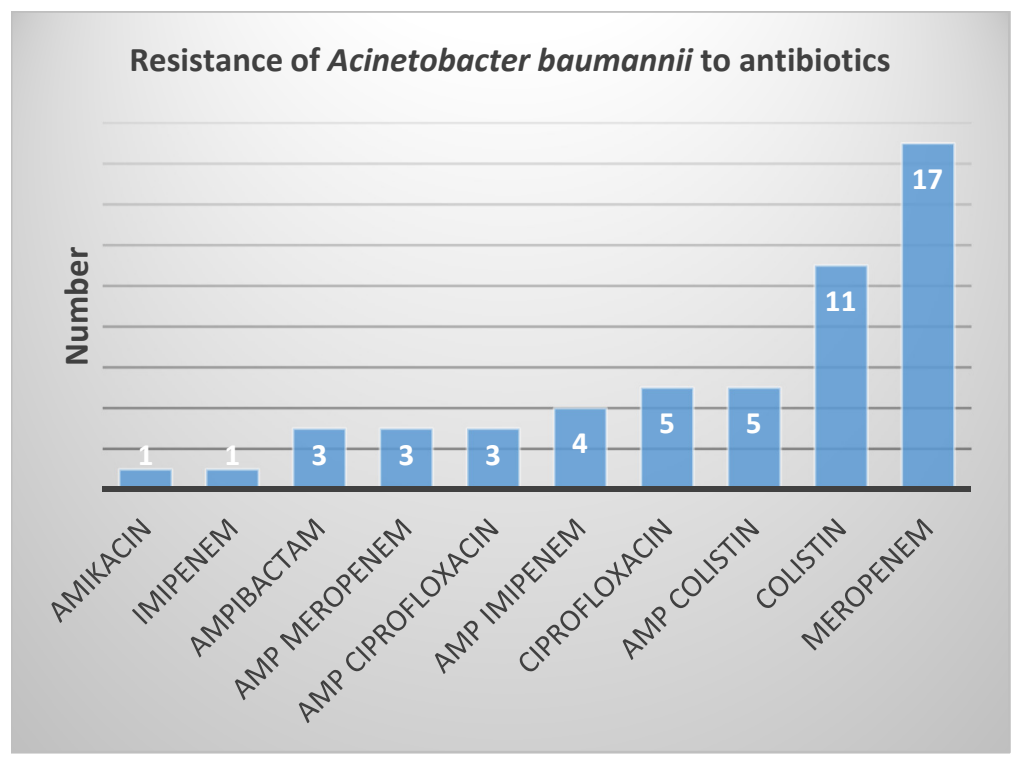

Fig. 1. The resistance frequency of Acinetobacter baumannii to antibiotics is shown 
baumannii in the ICU was 3 days, while the longest LOS was 98 days. The largest percentage of patients aged 6070 years $(22.1 \%)$, and a small percentage of patients aged $10-20$ years $(0.9 \%)$. Underlying diseases of the patients with MDR A. baumannii analyzed were (CABG [coronary artery bypass grafting], congenital heart disease, AVR [aortic valve replacement surgery], MVR [mitral valve replacement surgery], TVR [tricuspid valve replacement surgery], and PA [pulmonary artery] banding).

Of 23 patients with CABG, blood culture $(n=12,52.2 \%)$ had the highest frequency, of 19 patients with congenital heart disease (CHD), blood culture $(n=13,68.4 \%)$ had the highest frequency, and the lowest frequency belonged to urine culture $(\mathrm{n}=0,0.0 \%)$. Among 113 patients with MDR A. baumannii, 4 individuals associated with AVR showed a positive blood culture. Of 16 patients with MVR, sputum culture $(n=8,50 \%)$ had the highest frequency and the lowest frequency was belonged to CVC (central venus catheter culture $)(n=0,0.00 \%)$. Among female patients, the highest frequency was related to blood culture $(n=28$, $50.9 \%)$ and sputum culture $(n=27,49.1)$, respectively. However, urine culture and CVC had the lowest frequencies, respectively. Among male patients, the highest frequency was related to sputum culture $(n=26$, 44.8).

Patients in 2 age-groups (younger than 10 years and 40 50 years) had the highest frequency of BC. Patients in 2 age-groups (30-40 and 40-50 years) had the highest frequency of CVC. Patients in 3 age-groups (less than 10 years, 60-70 years, and 70-80 years) had CVC infection. Patients in 2 age-groups (20-30 and 50-60 years) showed signs of CAC (central artery catheter culture) infection. Among female patients, the highest frequency was related to $\mathrm{CVC}$ infection $(\mathrm{n}=58,9.1 \%)$. Among male patients, the highest frequency was related to CVC infection $(n=2$, $3.4 \%)$. Also, 10 (18.2\%) deaths were observed among 55 male patients, and $13(22.4 \%)$ deaths were reported among 58 female patients. Among age groups, the highest frequency of death was related to 30-40 and 70-80 age groups. The highest rates of antibiotic sensitivity were related to Meropenem 20 (17.7\%) and Colistin 16 (14.1\%) (Table 1).

\section{Discussion}

Infections due to A. baumannii and the development of MDR species are a major global problem threatening the public health (10). Management of MDR Acinetobacter spp. is of high importance for the treatment of different infections. Acinetobacter has natural antibiotic resistance and survives in hospitals and persist on different surfaces due to its ability to natural resistance genes. There are several possible or definite mechanisms by which this resistance occurs: (1) antimicrobial-inactivating enzymes; (2) decreased availability of bacterial targets, owing to reduced outer membrane permeability which occurs by the loss or decreased expression of porins and overexpression of multidrug efflux pumps; and (3) new mutations that alter cellular functions or the targets (changes in penicillin-binding proteins). A mix of these mechanisms may be found in the same bacterium $(20,21)$. This study evaluated the prevalence, antibiotic susceptibility, and the outcomes of the patients with MDR-A. baumannii in postcardiac surgery ICUs of Rajaei hospital. In this regard, in this study, it was observed that the prevalence rate of MDR- $A$. baumannii was $28.3 \%$ and the highest rates of antibiotic sensitivity were related to Meropenem and Colistin.

Nosocomial infection is referred to as an infection that has occurred within 72 hours, 3 days of discharge, or a 30day period after the surgery (22). About $30 \%$ of these infections are caused by Gram-negative bacteria and these statistics reach to $70 \%$ in ICU-hospitalized patients $(6$, 23). The incidence of nosocomial infections due to the MDR Gram-negative organisms, including Pseudomonas aeruginosa, A. bumanni (24), and positive germs such as methicillin-resistant Staphylococcus aureus (25), have increased dramatically in recent years. Castilho et al reported that the prevalence of MDR A. baumannii was elevated among isolates from the patients in 5 ICUs in Brazil. They also declared that most of $A$. baumannii isolates showed resistance to beta-lactam antimicrobial agents, particularly, carbapenems and third and fourthgeneration Cephalosporins. In addition, 91.1\% of the strains were MDR (26). However, the prevalence

Table 1. The resistance frequency of Acinetobacter baumannii to antibiotics and time of consumption

\begin{tabular}{lcc}
\hline Antibiotic & First round consumption & Total consumption within 6 months \\
\hline Amikacin & 1 & 5 \\
Ampicillin amikacin & 0 & 2 \\
Ampibactam & 3 & 11 \\
Ampicillin ampibactam & 0 & 1 \\
Meropenem & 17 & 44 \\
Ampicillin Meropenem & 3 & 9 \\
Imipenem & 1 & 4 \\
Ampicillin Imipenem & 4 & 10 \\
Gentamicin & 0 & 1 \\
Ampicillin gentamicin & 0 & 1 \\
Cotrimoxazole & 0 & 5 \\
Ampicillin Cotrimoxazole & 0 & 1 \\
Ciprofloxacin & 5 & 26 \\
Amp ciprofloxacin & 3 & 11 \\
Colistin & 11 & 63 \\
Ampicillin Colistin & 5 & 18 \\
Tazocin & 0 & 2 \\
Ampicillin Tazocin & 0 & 3 \\
\hline
\end{tabular}


reported in this study was lower than the other studies, including Dorodgar et al (63.2\%) (27) and RodríguezBaño et al (47\%) (28), but it was higher than the study of Javanbakht et al (22.6\%) (29). Two recent studies showed that A. baumannii strains were completely resistant to Meropenem $(17,27)$. In contrast, this study indicated that Meropenem and Colistin were the best choices for the treatment of patients in postcardiac surgery ICU. Colistin is the only therapeutic strategy for the treatment of carbapenem-resistant $A$. baumannii isolates. In a study in the USA, it was shown that the percentage of resistance to carbapenems in A. baumannii isolates increased from $21 \%$ in 2003-2005 to 48\% in 2009-2012 (13). Several studies have shown that $A$. baumannii isolates retained their sensitivity to Colistin. In addition, the prescription of Colistin or Tigecycline, solely or in combination, has the ability to reduce the risk of mortality (30). However, $A$. baumannii has the potential to be resistant to both Colistin (31) and Tigecycline (32). Therefore, precise diagnosis using antibiogram according to up-to-date standards may prevent the development of resistant isolates and help to reduce the burden of nosocomial infections. Based on various studies, the mortality rate related to nosocomial infections due to Acinetobacter species is approximately $7.8 \%$ to $23 \%(27,33)$. The reason for this increasing incidence of MDR strains in ICUs may be the severity of underlying conditions, the excessive use of antimicrobials, lower hygiene, and inappropriate disinfection strategies to control the infections in ICU (10). In addition, several genetic properties may contribute to antibiotic resistance such as antibiotic efflux, enzymatic inactivation (eg, AmpC and OXA-like $\beta$-lactamases) and reduced permeability of the outer membrane. Hood et al reported that antibiotic efflux effectively contributes to $\mathrm{NaCl}$ induced tolerance to Levofloxacin and Amikacin, whereas different mechanisms are involved in inducing the tolerance to Imipenem and Colistin. $\mathrm{NaCl}$ mediates tolerance to Colistin in MDR A. baumannii isolates. However, this study did not evaluate the concentrations of $\mathrm{NaCl}$ in the specimens (34).

Ayyıldız et al indicated that $8.8 \%$ of the patients in the ICU had $A$. baumannii when they were using extracorporeal membrane oxygenation (ECMO) as a standard technique to support temporary cardiovascular and respiratory aid to the patients in ICU (35). Moreover, Lee et al showed that $7.5 \%$ of the patients were colonized with MDR A. baumannii. Compared to uncolonized patients, the colonized patients presented an elevated mortality rate; also, the ICU length of stay (LOS) was significantly higher in colonized patients than in uncolonized patients (36). Based on the data of this study, the shortest LOS for patients with MDR A. baumannii in the ICU was 3 days, while the longest LOS was 98 days.

In a study conducted by Pérez-Pedrero et al, it was concluded that the treatment with Colistin inhalation would improve the results in patients with pulmonary infections of the A. baumannii. They also stated that Colistin inhalation should be used as an adjuvant therapy with intravenous therapy in patients with acute respiratory infection caused by A. baumannii (37). In another study conducted by Chan et al on 55 patients, it was revealed that pneumonia associated with ventilator in Carbapenemresistant $A$. baumannii can be effectively treated with second-line drugs. However, it is unclear whether combined therapy has a better effect (9). Joung et al performed a study over a period of 6 years in 116 patients with Acinetobacter-acquired pneumonia. They reported that inappropriate antimicrobial treatment for $A$. baumannii has been associated with adverse outcomes. Physicians should be aware that appropriate antibacterial therapy must be prescribed immediately to reduce the risk of adverse outcomes if $A$. baumannii is isolated from respiratory specimens of patients (38). In a study conducted by Erbay et al on 103 patients with bloodassociated infections due to A. baumannii, the delay in receiving proper treatment resulted in poor clinical outcomes in the patients. The use of more severe empirical treatment can be considered in hospitals where A. baumannii isolates have high resistance (39). This study had several limitations. First, it was not possible to provide documents to describe resistance mechanisms. A better understanding of possible mechanisms may help prevention and control approaches. Second, it is hard to generalize the findings to all areas of other wards or regions. Third, this was a cross sectional study and further investigation is needed at other hospitals and wards to follow up the alterations in resistance status of this microorganism.

\section{Conclusion}

This study presented an increased prevalence of antibiotic-resistant Acinetobacter baumannii strains in ICU. The findings demonstrated that A. baumannii strains isolated from postcardiac surgery ICUs had a high prevalence and sensitivity to Meropenem and Colistin. However, more precise and accurate culture methods and molecular techniques are required to monitor nosocomial infections. Therefore, the treatment of the patients hospitalized in ICUs may be more achievable by prescribing appropriate antibiotic therapy and infection control policies by precise disinfection strategies.

\section{Conflict of Interests}

The authors declare that they have no competing interests.

\section{References}

1. Howard A, O'Donoghue M, Feeney A, Sleator RD. Acinetobacter baumannii: an emerging opportunistic pathogen. Virulence. 2012;3(3):243-50.

2. Perez F, Hujer AM, Hujer KM, Decker BK, Rather PN, Bonomo RA. Global challenge of multidrug-resistant Acinetobacter baumannii. Antimicrob Agents Chemother. 2007;51(10):3471-84.

3. Mansouri D, Mekaniki N, Kamali M, Ghafourian A, Marjani M, Mohammad TZ. A young dentist with fever, mediastinal lymphadenopathies and pulmonary infiltration (photo quiz). Tanaffos J. 2009;8(3):77-80.

4. Eveillard M, Soltner C, Kempf M, Saint-André JP, Lemarié C, Randrianarivelo $\mathrm{C}$, et al. The virulence variability of different Acinetobacter baumannii strains in experimental pneumonia. J Infect. 2010;60(2):154-61.

5. Metan G, Sariguzel F, Sumerkan B. Factors influencing survival in 
patients with multi-drug-resistant Acinetobacter bacteraemia. Eur J Intern Med. 2009;20(5):540-4.

6. Weinstein RA, Gaynes R, Edwards JR, System NNIS. Overview of nosocomial infections caused by gram-negative bacilli. Clin Infect Dis. 2005;41(6):848-54.

7. Eslami N, Tavakol M, Mesdaghi M, Gharegozlou M, Casanova JL, Puel A, et al. A gain-of-function mutation of STAT1: a novel genetic factor contributing to chronic mucocutaneous candidiasis. Acta Microbiol Immunol Hung. 2017;64(2):191-201.

8. Montefour K, Frieden J, Hurst S, Helmich C, Headley D, Martin M, et al. Acinetobacter baumannii: an emerging multidrug-resistant pathogen in critical care. Criti Care Nurse. 2008;28(1):15-25.

9. Chan JD, Graves JA, Dellit TH. Antimicrobial treatment and clinical outcomes of carbapenem-resistant Acinetobacter baumannii ventilatorassociated pneumonia. J Intensive Care Med. 2010;25(6):343-8.

10. Wong D, Nielsen TB, Bonomo RA, Pantapalangkoor P, Luna B, Spellberg B. Clinical and pathophysiological overview of Acinetobacter infections: a century of challenges. Clin Microbiol Rev. 2017;30(1):409-47.

11. Baharlou R, Atashzar MR, Vasmehjani AA, Rahimi E, Khoshmirsafa $\mathrm{M}$, Seif F, et al. Reduced levels of T-helper 17-associated cytokines in the serum of patients with breast cancer: indicators for following the course of disease. Cent Eur J Immunol. 2016;41(1):78.

12. Mohsenzadegan M, Seif F, Farajollahi MM, Khoshmirsafa M. Antioxidants as chemopreventive agents in prostate cancer: A gap between preclinical and clinical studies. Recent Pat Anticancer Drug Discov. 2018;13(2):224-39.

13. Zilberberg MD, Kollef MH, Shorr AF. Secular trends in Acinetobacter baumannii resistance in respiratory and blood stream specimens in the United States, 2003 to 2012: a survey study. J Hosp Med. 2016;11(1):21-6.

14. Kanafani Z, Kanj S. Acinetobacter infection: Epidemiology, microbiology, pathogenesis, clinical features, and diagnosis. Wolters Kluwer. 2013;2:21-33.

15. Forbes BA, Sahm DF, Weissfeld AS. Study Guide for Bailey and Scott's Diagnostic Microbiology-E-Book: Elsevier Health Sciences; 2016.

16. Patel J, Cockerill F, Bradford P, Eliopoulos G, Hindler J, Jenkins S, et al. Clinical and Laboratory Standards Institute. Performance standards for antimicrobial susceptibility testing Clinical and Laboratory Standards Institute, USA. 2016.

17. Kazemi H, Yadegarynia D, Roodsari SR, Arab-Mazar Z. Evaluation of Antimicrobial Susceptibility Among Acintobacter baumannii by ETest Method at Khatam-Al-Anbia Hospital During 2013-2015. Zahedan J Res Med Sci. 2017;19(1).

18. Paterson DL. The epidemiological profile of infections with multidrug-resistant Pseudomonas aeruginosa and Acinetobacter species. Clin Infect Dis. 2006;43(Supplement 2):S43-S8.

19. Seif F, Ghalehbaghi B, Aazami H, Mohebbi A, Ahmadi A, Falak R, et al. Frequency of CD4+ and CD8+ T cells in Iranian chronic rhinosinusitis patients. Allergy Asthma Clin Immunol. 2018;14(1):47.

20. Manchanda V, Sanchaita S, Singh N. Multidrug resistant acinetobacter. J Global Infect Dis. 2010;2(3):291.

21. Rekabi M, Arshi S, Bemanian MH, Rekabi V, Rajabi A, Fallahpour $\mathrm{M}$, et al. Evaluation of a new protocol for wheat desensitization in patients with wheat-induced anaphylaxis. Immunotherapy. 2017;9(8):637-45.

22. Robinson T, Bu D, Carrique-Mas J, Fèvre E, Gilbert M, Grace D, et al. Antibiotic resistance is the quintessential One Health issue. Trans R Soc Trop Med Hyg. 2016;110(7):377-80.

23. Seif F, Khoshmirsafa M, Mousavi M, Beshkar P, Rafeian-Kopaei M, Bagheri N, et al. Interleukin-21 receptor might be a novel therapeutic target for the treatment of rheumatoid arthritis. J Exp Clin Med. 2014;6(2):57-61.

24. Crandon JL, Kuti JL, Jones RN, Nicolau DP. Comparison of 2002 2006 OPTAMA programs for US hospitals: focus on gram-negative resistance. Ann Pharmacother. 2009;43(2):220-7.

25. Dancer SJ. Importance of the environment in meticillin-resistant Staphylococcus aureus acquisition: the case for hospital cleaning. Lancet infect Dis. 2008;8(2):101-13.

26. Castilho SRA, de Miranda Godoy CS, Guilarde AO, Cardoso JL, André MCP, Junqueira-Kipnis AP, et al. Acinetobacter baumannii strains isolated from patients in intensive care units in Goiânia, Brazil: Molecular and drug susceptibility profiles. PloS One. 2017;12(5):e0176790.
27. Dorodgar S, Hatami H, Yadegarynia D, Arab-Mazar Z. Nosocomial Infections: Multicenter Surveillance of Antimicrobial Resistance in Tehran During 2015-2017. Arch Clin Infect Dis. 2018(In Press).

28. Rodríguez-Baño J, Cisneros JM, Fernández-Cuenca F, Ribera A, Vila J, Pascual A, et al. Clinical features and epidemiology of Acinetobacter baumannii colonization and infection in Spanish hospitals. Infect Control Hosp Epidemiol. 2004;25(10):819-24.

29. Javanbakht A, Askari E, Danesh L, Moghadas N, Mostafavi I, Naderinasab M. The incidence of cross infections in Imam Reza hospital, Mashhad, Iran. Iran J Microbiol. 2012;4(4):177.

30. Sukanya S, Lakshmi V, Padmaja K. Prevalence of multidrug resistant Acinetobacter baumannii in clinical samples in a tertiary care hospital. Int J Infect Control. 2014;11:3.

31. Tan CH, Li J, Nation RL. Activity of colistin against heteroresistant Acinetobacter baumannii and emergence of resistance in an in vitro pharmacokinetic/pharmacodynamic model. Antimicrob Agents Chemother. 2007;51(9):3413-5.

32. Deng M, Zhu MH, Li JJ, Bi S, Sheng ZK, Hu FS, et al. Molecular epidemiology and mechanisms of tigecycline resistance in clinical isolates of Acinetobacter baumannii from a Chinese university hospital. Antimicrob Agents Chemother. 2014;58(1):297-303.

33. Mardani M, Tabarsi P, Yadegarinia D, Talebi Taher M, Najafi N, Hajabdolbaghi $M$, et al. Treatment of invasive fungal infection: Recommendations from scientific leaders' meeting on November 3rd, 2011 tehran-iran. Iran J Clin Infect Dis. 2011;6(4).

34. Hood MI, Jacobs AC, Sayood K, Dunman PM, Skaar EP. Acinetobacter baumannii increases tolerance to antibiotics in response to monovalent cations. Antimicrob Agents Chemother. 2010;54(3):1029-41.

35. Ayyıldız P, Kasar T, Ozturk E, Yildiz O, Ozturk S, Ergul Y, et al. The Evaluation of Nosocomial Infections in Pediatric Patients with Extracorporeal Membrane Oxygenation Support. Braz J Cardiovasc Surg. 2017;32(6):468-74.

36. Lee H, Lee H. Clinical and economic evaluation of multidrugresistant Acinetobacter baumannii colonization in the intensive care unit. Infect Chemother. 2016;48(3):174-80.

37. Pérez-Pedrero M, Sánchez-Casado M, Rodríguez-Villar S. Nebulized colistin treatment of multi-resistant Acinetobacter baumanni pulmonary infection in critical ill patients. Med Intensiva (English Edition). 2011;35(4):226-31.

38. Joung MK, Kwon KT, Kang CI, Cheong HS, Rhee JY, Jung DS, et al. Impact of inappropriate antimicrobial therapy on outcome in patients with hospital-acquired pneumonia caused by Acinetobacter baumannii. J Infect. 2010;61(3):212-8.

39. Erbay A, İdil A, Gözel MG, Mumcuoğlu İ, Balaban N. Impact of early appropriate antimicrobial therapy on survival in Acinetobacter baumannii bloodstream infections. Int $\mathrm{J}$ Antimicrob Agents. 2009;34(6):575-9. 